\title{
Delayed stenosis as a consequence of angioplasty for subarachnoid hemorrhage-induced vasospasm
}

\author{
Case report
}

\author{
Akeel Merchant, Doniel Drazin, M.A., John Dalfino, M.D., ${ }^{1}$ \\ Junichi Yamamoto, M.D., Ph.D., and Alan S. Boulos, M.D.
}

Division of Neurosurgery, Albany Medical Center, Albany, New York

\begin{abstract}
The authors report a case of restenosis in the bilateral internal carotid arteries (ICAs) following angioplasty for cerebral vasospasm. This 53-year-old woman suffering subarachnoid hemorrhage due to a ruptured posterior communicating artery aneurysm had severe vasospasm and underwent angioplasty of the left and right ICAs and middle cerebral arteries. Two months later, a follow-up CT angiogram revealed bilateral ICA stenoses.

Transluminal angioplasty leads to long-term connective tissue damage in the medial and adventitial layers from the disruption of the arrangement of collagen fibers due to stretching and tearing, resulting in loss of transmission of contractile forces. Furthermore, following endothelial cell denudation and stretching and rupture of internal elastic lamina from angioplasty, reendothelialization of the intimal layer composed of smooth muscle cells may also explain the contractile properties of restenosis. Other factors such as macrophage-induced inflammation and reactive oxygen species accumulation may also contribute to restenosis. This is the second reported case of restenosis following angioplasty to treat vasospasm, although restenosis is a known complication of angioplasty for treatment of atherosclerosis. In addition, this is the first case of restenosis in the bilateral ICAs following angioplasty for vasospasm. This report presents an illustrative case study and reviews the pathophysiology of angioplasty and restenosis. (DOI: 10.3171/2009.2.FOCUS0912)
\end{abstract}

KEY WORDS • vasospasm • angioplasty • internal carotid artery $\bullet \quad$ aneurysm

A NGIOPLASTY was first used in 1977 for dilating atherosclerotic plaques in coronary arteries. $.^{5} \mathrm{Al}-$ though restenosis after angioplasty is a common occurrence in atherosclerosis cases, ${ }^{12}$ it has only been reported once after the use of angioplasty for cerebral vasospasm. Sedet et al. ${ }^{11}$ reported delayed stenosis of middle and anterior cerebral arteries. In this paper, we report delayed stenosis of the ICAs. To our knowledge, this is the second reported case of delayed stenosis following angioplasty for treatment of cerebral vasospasm.

\section{Case Report}

This 53-year-old woman suffered severe SAH (Hunt and Hess Grade IV) due to a ruptured posterior communicating artery aneurysm. Initial CT angiography revealed widely patent ICAs and an associated right posterior communicating aneurysm. The patient underwent

\footnotetext{
Abbreviations used in this paper: ICA = internal carotid artery; $\mathrm{SAH}=$ subarachnoid hemorrhage.
}

a frontotemporal craniectomy on an emergency basis and clip ligation of the aneurysm and evacuation of the intracerebral hematoma were performed. Seven days after admission, CT angiography revealed severe diffuse vasospasm. As a result, angioplasty of the left and right ICAs and middle cerebral arteries was conducted with a Hyperglide balloon (ev3, Inc.). After the angioplasty procedure, verapamil was infused intraarterially to dilate more distal branches. Angioplasty of the right $\mathbf{M}_{1}$ and $\mathrm{M}_{2}$ segments was then performed after exchanging the microcatheter for a Maverick balloon catheter (Boston Scientific Corp.). Bone plate replacement was performed 2 weeks later. The following week, the patient underwent placement of a ventriculoperitoneal shunt on her left side due to hydrocephalus resulting from $\mathrm{SAH}$. At 1-month follow-up, the patient exhibited signs of fatigue, diminished vision in both eyes, and short-term memory loss.

Despite continual fatigue and short-term memory loss at 1 month follow-up, the patient improved neurologically and had no focal neurological symptoms at 3 months. A follow-up CT angiogram obtained 6 months postoperatively revealed bilateral ICA stenoses that were 

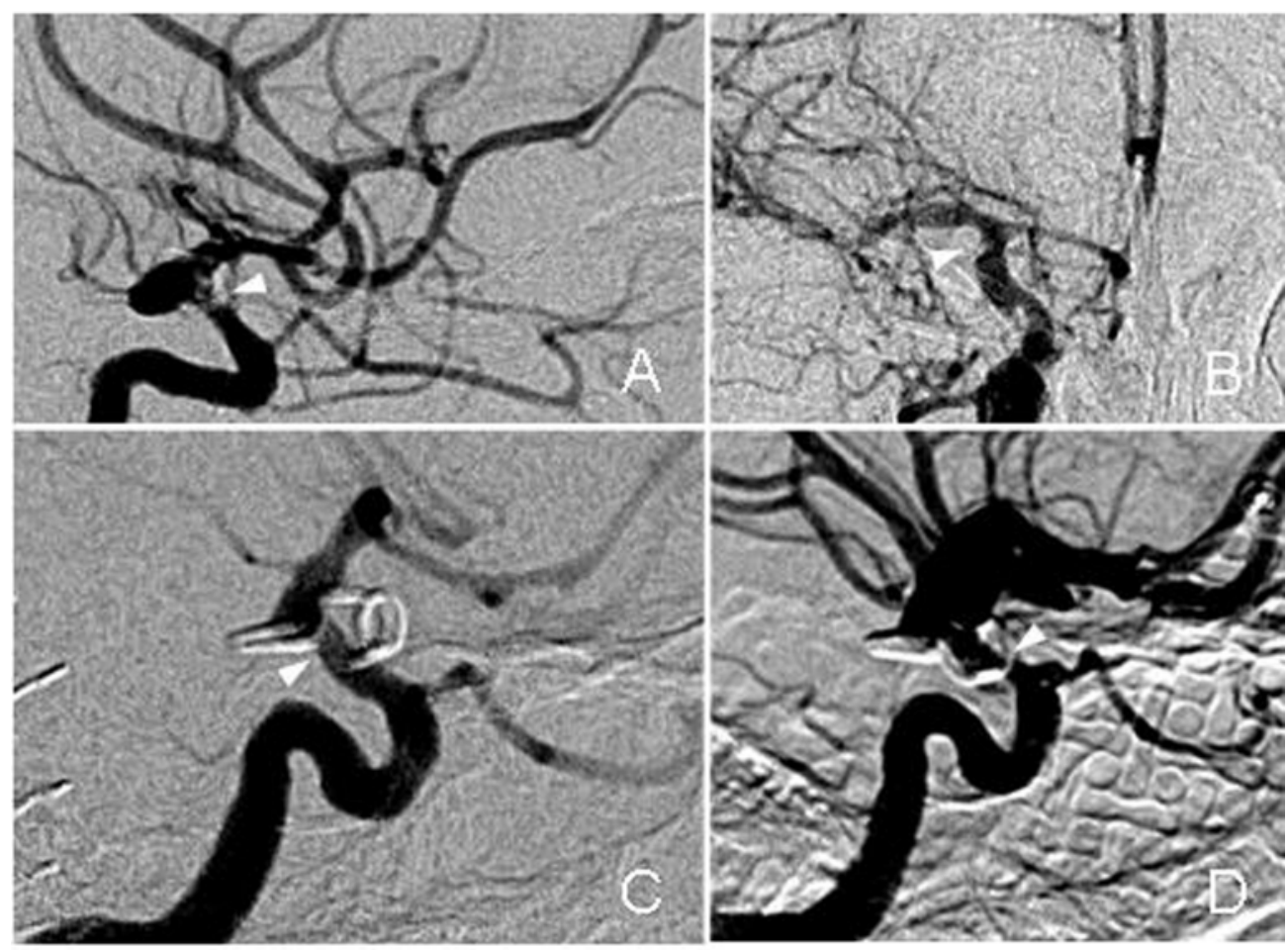

FIG. 1. Selective right ICA arteriograms obtained in a 53-year-old patient with vasospasm following rupture of a right posterior carotid wall aneurysm. Pretreatment lateral (A) and anteroposterior (B) projections show moderate stenosis of the supraclinoid ICA (arrowheads). Following balloon angioplasty (C), the lumen of the vessel is restored to normal (arrowhead). A follow-up angiogram obtained 9 months later (D) shows recurrent stenosis (arrowheads) of the supraclinoid ICA.

especially prominent in the supraclinoid region. A CT perfusion study suggested diminished perfusion bilaterally. Repeat diagnostic cerebral angiography 9 months after angioplasty confirmed persistent stenosis in bilateral ICAs (Figs. 1 and 2).

\section{Discussion}

Vasospasm is common in patients with aneurysmal SAH. It is a leading cause of cerebral ischemia, and hence death and injury after an aneurysm rupture. ${ }^{6} \mathrm{Ap}$ proximately $50 \%$ of SAH cases result in death while $15 \%$ result in disability. ${ }^{4}$ Research suggests that nitric oxide, endothelin-1 and bilirubin oxidation products, and inflammation are important contributors to cerebral vasospasm. ${ }^{4}$

Angioplasty, although initially used in coronary atherosclerosis, was first shown to be an effective treatment for vasospastic arteries in 1984 by Zubkov et al. ${ }^{15}$ It works by dilating the constricted arteries via a balloon catheter and thus preventing ischemic conditions. Clinical studies by Newell et al. ${ }^{8}$ and Bejjani et al. ${ }^{2}$ have alluded to its efficacy and safety. However, the case report presented here, being only the second reported case of restenosis after cerebral vasospastic angioplasty, suggests that there can be delayed effects of angioplasty for the treatment of vasospasm. This finding is important as this effect may not be unique and continued surveillance imaging of patients who undergo angioplasty for vasospasm may be required. Indeed, we are considering bypass surgery or stent placement to restore normal cerebral blood flow in our patient, but given the unusual circumstances and the patient's continued neurological recovery, we have not decided on therapy yet.

\section{Pathophysiology}

A study by Chavez et al. ${ }^{3}$ has shown that transluminal angioplasty leads to endothelial cell denudation, stretching and rupture of internal elastic lamina, and changes in smooth muscle cells of the medial layer, but these changes resolved within 7 days. Yamamoto et al., ${ }^{14}$ however, showed long-term connective tissue damage in the medial and adventitial layers from the disruption of the arrangement of collagen fibers due to its stretching and tearing. Since extracellular matrix is an important medium in the transmission of contractile forces, its disruption may explain the occurrence of delayed stenosis.

A study by Wakayama et al. ${ }^{13}$ investigated the pathophysiology of restenosis with the use of a transluminal vascular injury model in rodents. The idea behind the use of this model was to create a suture with a diameter larger than that of a rat's ICA, such that the vessel would be dilated when the suture was advanced. Specifically, a 25-mm length of 3-0 surgical suture was modified with an epoxide resin-coated region at the tip, $0.6 \mathrm{~mm}$ in diameter and $5 \mathrm{~mm}$ in length. For 1 minute, the length of suture material was inserted into the external carotid artery and advanced $18-20 \mathrm{~mm}$ into the ICA, leading to rapid enlargement of the ICA (normal luminal diameter is $0.3 \mathrm{~mm}) .{ }^{9}$ Following injury using this intraluminal suture technique, there was loss of endothelial cells and the 


\section{Postangioplasty stenosis}

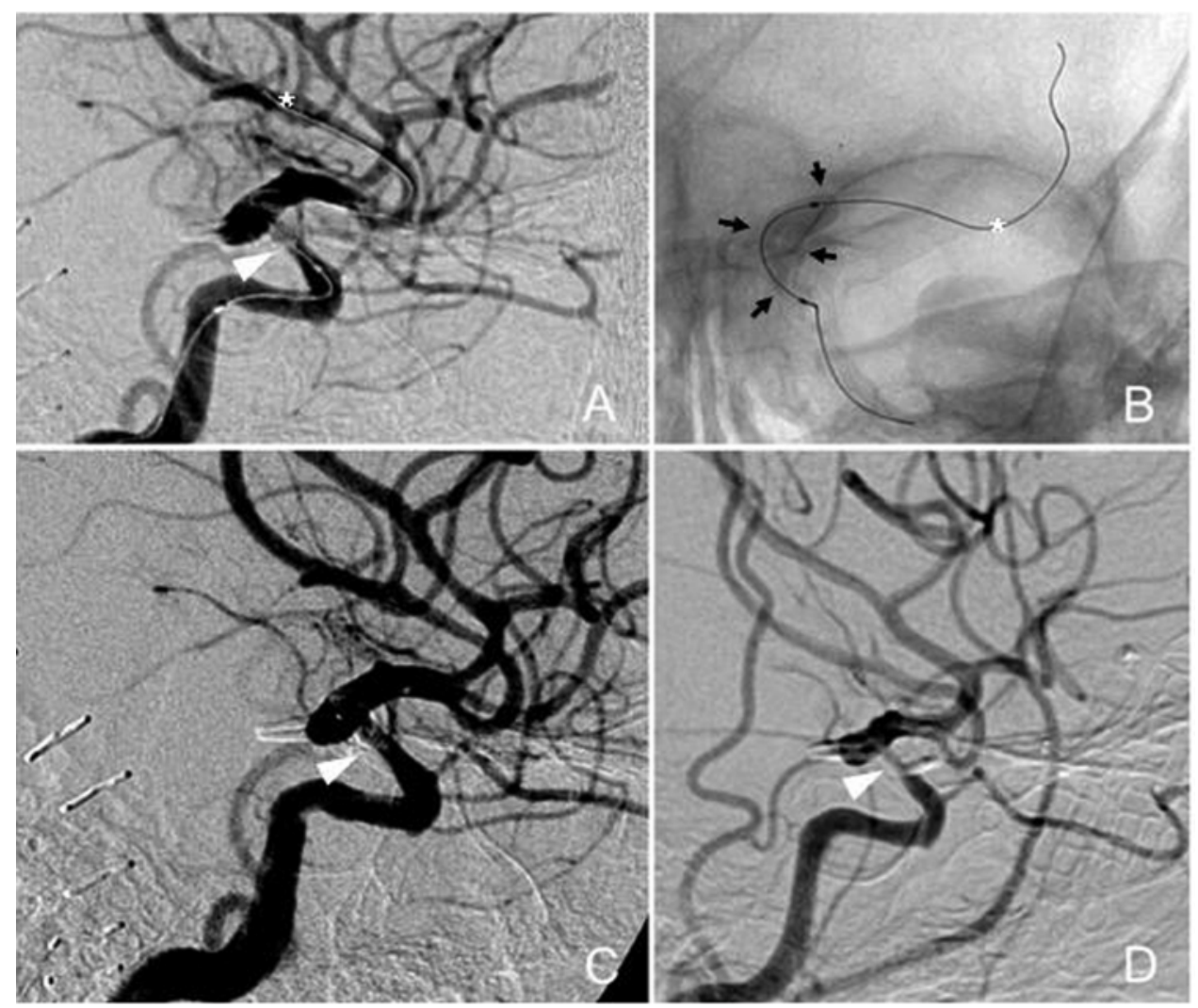

FIG. 2. Pretreatment and posttreatment left ICA angiograms. The pretreatment angiogram shows vasospasm of the supraclinoid segment of the left ICA (A, arrowhead). An X-pedion 0.010" guidewire (ev3, Inc.) was advanced through the stenotic segment and into the middle cerebral artery (white asterisk). Inflation of a contrast-filled HyperGlide balloon (B, small black arrows) within the supraclinoid segment dilated the vessel lumen, improving flow (C, arrowhead). A follow-up angiogram obtained 9 months later, however, shows restenosis of the supraclinoid segment of the left ICA (D, arrowhead).

smooth muscle cells of the medial layer. However between 1 and 4 weeks afterward there was a reemergence of the neointimal layer composed of smooth muscle cells and the surrounding extracellular matrix. This new intimal constitution may explain the contractile properties of restenosis.

Rubin et al..$^{10}$ explained that the occurrence of restenosis may be mitigated by inflammation. They showed that within 24 hours after injury macrophages begin migrating into the adventitial layer. Within 1 week, they spread to the medial and intimal layers but were more prominent in the latter. Neointimal hyperplasia was evident within 3 weeks, during which time the presence of macrophages could also be detected.

Finally, injury to blood vessels as a result of angioplasty can lead to activation of reactive oxygen species at the injury site from molecular oxygen. One such reactive oxygen species, the superoxide radical, exerts numerous effects such as inactivation of endothelium-derived relaxing factor, lipid hydroperoxide-mediated modulation of vascular tone, and platelet activation. ${ }^{7}$ Although generated as part of a repair reaction, these effects can lead to occurrence of restenosis. ${ }^{1}$

\section{Applicability of This Report}

Angioplasty for vasospasm has been an important adjunct for the treatment of cerebral ischemia in the setting of SAH. Though the efficacy of the procedure has not been proven with Class I evidence, it is thought to be effective if administered within a short time after ischemic symptoms develop. This report simply reminds treating physicians that there can be delayed consequences to this therapy and follow-up surveillance imaging is important in patients who have undergone this treatment for vasospasm.

In addition, the use of the compliant softer balloons may play a role in the development of delayed stenosis. In both our case and the previously reported case, Hyperglide balloons were used. These soft balloons inflate to between 3 and $5 \mathrm{~mm}$ in diameter and so may stretch the vessel beyond its normal size. The advantage of this technique is that the vessel appears to be restored to normal size immediately and rarely requires retreatment for vasospasm at the same site, but the potential disadvantage is that stretching the vessel beyond its normal diameter may instigate the above-proposed mechanisms leading to the development of delayed stenosis. While noncompliant coronary balloons can also be used to treat vasospasm, the diameter of the balloon is selected to be smaller than the normal vessel (for example, for a 3-mm ICA, a 2.5-mm-diameter balloon may be selected). Hence, when the balloon is fully inflated the vessel is not stretched be- 
yond its normal diameter. One potential risk associated with these types of balloons is the chance of vasospasm recurring in the vessel after treatment. If the chosen balloon diameter is too small (for example, if a $1.5-\mathrm{mm}$ balloon were to be selected in the above example) then there remains a chance the vessel will not be stretched enough and repeated vasospasm may occur during the acute period. The noncompliant balloons are also stiffer, so they may be harder to track to the affected vessel. In addition, they tend to straighten the artery, so if the balloon is too long, it may kink or injure the treated vessel. In this reported case, we used both kinds of balloons, and the delayed stenosis only occurred in the area treated by means of the compliant balloons.

\section{Conclusions}

We report the unique finding of delayed stenosis following angioplasty of vasospastic ICAs using compliant balloons. The stenoses occurred bilaterally at the same site. Fortunately, there were no focal neurological symptoms attributable to these stenoses in our patient, but this case report should alert practitioners of this potential complication. Surveillance imaging is important after angioplasty for vasospasm and should be performed in a delayed fashion. Treatment of the delayed stenosis could include stent placement or intracranial bypass procedures.

\section{Disclaimer}

The authors report no conflict of interest concerning the materials or methods used in this study or the findings specified in this paper.

\section{References}

1. Azevedo LC, Pedro MA, Souza LC, de Souza HP, Janiszewski M, da Luz PL, et al: Oxidative stress as a signaling mechanism of the vascular response to injury: the redox hypothesis of restenosis. Cardiovasc Res 47:436-445, 2000

2. Bejjani GK, Bank WO, Olan WJ, Sekhar LN: The efficacy and safety of angioplasty for cerebral vasospasm after subarachnoid hemorrhage. Neurosurgery 42:979-987, 1998

3. Chavez L, Takahashi A, Yoshimoto T, Su CC, Sugawara T, Fujii Y: Morphological changes in normal canine basilar arteries after transluminal angioplasty. Neurol Res 12:12-16, 1990
4. Crowley RW, Medel R, Kassell NF, Dumont AS: New insights into the causes and therapy of cerebral vasospasm following subarachnoid hemorrhage. Drug Discov Today 13:254-260, 2008

5. Gruntzig A: Transluminal dilatation of coronary-artery stenosis. Lancet 1:263, 1978

6. Kassell NF, Sasaki T, Colohan AR, Nazar G: Cerebral vasospasm following aneurysmal subarachnoid hemorrhage. Stroke 16:562-572, 1985

7. Laurindo FRM, Daluz PL, Uint L, Rocha TF, Jaeger RG, Lopes EA: Evidence for superoxide radical-dependent coronary vasospasm after angioplasty in intact dogs. Circulation 83:1705-1715, 1991

8. Newell DW, Eskridge JM, Mayberg MR, Grady MS, Winn HR: Angioplasty for the treatment of symptomatic vasospasm following subarachnoid hemorrhage. J Neurosurg 71:654660,1989

9. Ono S, Date I, Onoda K, Ohmoto T: Time course of the diameter of the major cerebral arteries after subarachnoid hemorrhage using corrosion cast technique. Neurol Res 25:383-389, 2003

10. Rubin P, Williams JP, Riggs PN, Bartos S, Sarac T, Pomerantz R, et al: Cellular and molecular mechanisms of radiation inhibition of restenosis. Part I: role of the macrophage and platelet-derived growth factor. Int J Radiat Oncol Biol Phys 40:929-941, 1998

11. Sedat J, Chau Y, Popolo M, Gindre S, Rami L, Orban JC: Restenosis after balloon angioplasty for cerebral vasospasm. Cardiovasc Intervent Radiol 32:337-340, 2009

12. Siddiq F, Vazquez G, Memon MZ, Suri MF, Taylor RA, Wojak $\mathrm{JC}$, et al: Comparison of primary angioplasty with stent placement for treating symptomatic intracranial atherosclerotic diseases: a multicenter study. Stroke 39:2505-2510, 2008

13. Wakayama K, Shimamura M, Sata M, Koibuchi N, Sato N, Ogihara T, et al: A model of cerebrovascular injury in rats. J Neurosci Methods 175:187-195, 2008

14. Yamamoto Y, Smith RR, Bernanke DH: Mechanism of action of balloon angioplasty in cerebral vasospasm. Neurosurgery 30:1-6, 1992

15. Zubkov YN, Nikiforov BM, Shustin VA: Balloon catheter technique for dilatation of constricted cerebral arteries after aneurysmal SAH. Acta Neurochir (Wien) 70:65-79, 1984

Manuscript submitted January 15, 2009.

Accepted February 11, 2009.

Address correspondence to: Alan S. Boulos, M.D., Division of Neurosurgery, Albany Medical Center, 47 New Scotland Avenue, Albany, New York 12208. email: alan.boulos@gmail.com. 